\title{
The Impact of Maternal Asthma on the Preterm Infants' Gut Metabolome and Microbiome - The Microbiome, Atopic Disease, and Prematurity (MAP) Study
}

\section{Shiyu S. Bai-Tong}

University of California, San Diego

Megan S. Thoemmes

University of California, San Diego

Kelly C. Weldon

University of California, San Diego

Diba Motazavi

University of California, San Diego

Jessica Kitsen

University of California, San Diego

Shalisa Hansen

University of California, San Diego

Annalee Furst

University of California, San Diego

\section{Bob Geng}

University of California, San Diego

\section{Se Jin Song}

University of California, San Diego

Jack A. Gilbert

University of California, San Diego

\section{Lars Bode}

University of California, San Diego

Pieter C. Dorrestein

University of California, San Diego

Rob Knight

University of California, San Diego

Sydney A. Leibel

University of California, San Diego

Sandra L. Leibel ( $\sim$ saleibel@health.ucsd.edu )

University of California, San Diego 


\section{Research Article}

Keywords: asthma, prematurity, microbiome, metabolome

Posted Date: December 6th, 2021

DOI: https://doi.org/10.21203/rs.3.rs-1075590/v1

License: (c) (1) This work is licensed under a Creative Commons Attribution 4.0 International License. Read Full License 


\section{Abstract}

Preterm infants are at a greater risk for the development of asthma and atopic disease, which can lead to lifelong negative health consequences. This may, in part, be due to alterations that occur in the gut microbiome and metabolome during their stay in the Neonatal Intensive Care Unit (NICU). To explore the differential roles of family history (i.e., predisposition due to maternal asthma diagnosis) and hospitalrelated environmental and clinical factors that alter microbial exposures early in life, we looked at a unique cohort of preterm infants born $£ 34$ weeks gestational age from two local level III NICUs, as part of the MAP (Microbiome, Atopic disease, and Prematurity) Study. Weekly stool, milk feeds, and saliva were collected until hospital discharge, and monthly stool and milk samples were collected at home until one year of age. We also chose a sub-cohort of infants whose mothers had a history of asthma and matched gestational age and sex to infants of mothers without a history of asthma (control). We performed a prospective, paired metagenomic and metabolomic analysis of stool and milk feed samples collected at birth, 2 weeks, and 6 weeks postnatal age. Although there were clinical factors associated with shifts in the diversity and composition of stool-associated bacterial communities, maternal asthma diagnosis did not play an observable role in shaping the infant gut microbiome in the study period. There were significant differences, however, in the metabolite profile between the maternal asthma and control groups at 6 weeks postnatal age. The most notable changes occurred in the linoleic acid spectral network, which plays a role in inflammatory and immune pathways, suggesting early metabolomic changes in the gut of preterm infants born to mothers with a history of asthma. Our pilot analysis suggests that a history of maternal asthma alters a preterm infants' metabolomic pathways in the gut as early as the first 6 weeks of life.

\section{Introduction}

Asthma is an immune-mediated multifactorial disease that is influenced by both genetic and environmental factors. In particular, a maternal history of asthma is thought to increase the risk of asthma development in their children ${ }^{1,2}$. The composition of the gut microbiome, which is shaped by environmental exposure, is associated with the development of allergic diseases among children, likely due to its modulation of the innate and adaptive immune system ${ }^{3-6}$. For example, immature gut microbial composition at 1-year of age is associated with an increased risk of asthma by age five in the offspring of mothers with asthma ${ }^{7}$. Additionally, metabolites, the products of the metabolic pathway in biological systems which are reflective of genetic and environmental factors, play a critical role in disease pathogenesis and are implicated in common neonatal conditions and atopic diseases, such as asthma ${ }^{8-}$ ${ }^{10}$. However, the mechanisms by which gut-associated metabolites influence lung health remains unclear

11. Despite mounting evidence on the importance of genetic and environmental components in asthma pathogenesis, their relative effects are rarely studied together, and there have yet to be any studies that consider their combined importance in shaping both the microbiome and metabolome of the preterm infant gut over time. Therefore, the characterization of gut-associated bacteria and metabolites in 
response to these combined factors might allow us to more accurately evaluate childhood asthma risk in preterm infants.

The assembly of the microbiome in the infant gut represents a de novo microbial community influenced by various environmental factors ${ }^{12,13}$. These factors in healthy term infants include breastfeeding, contact with adults, whether they have older siblings in the home, and pet ownership ${ }^{14-17}$. Preterm infants experience dramatically different exposures compared to healthy term infants. Prior to delivery, preterm fetuses are commonly exposed to antenatal antibiotics and steroids, and preterm infants are delivered via C-section more frequently than term infants ${ }^{18}$. Postnatally, infants admitted to the NICU are exposed to antibiotics, have decreased contact with parents and siblings, and experience delays in the introduction of enteral and oral feeds. These factors have been shown to alter the gut microbiome, resulting in higher numbers of Enterobacteriaceae and Clostridiaceae, and the absence of Bifidobacteriaceae and Lactobacillaceae, a pattern consistent with dysbiosis ${ }^{14,19,20}$. Alterations to the gut microbiome are of particular concern among preterm infants, because dysbiosis increases the risk of allergic diseases like asthma ${ }^{20}$.

The intestinal microbiota interacts with the immune system, in part, through the production of metabolites, which can be taken up by immune and epithelial cells ${ }^{21,22}$. For example, short chain fatty acids, produced by a variety of microbes during fermentation of dietary fiber, are protective against food allergy and pulmonary allergic inflammation in mice ${ }^{23-26}$, and it has also been shown that polyunsaturated fatty acids (PUFA) interact with human microbes in asthma pathogenesis ${ }^{23}$. Understanding the relevant metabolomic mechanisms in gut microbiome-host interactions and dysbiosis would allow for new insights in the pathogenesis of childhood asthma and critical clinical applications.

To explore the relative impacts of prematurity and a family history of maternal asthma diagnosis on the gut microbiome and metabolome, we recruited preterm infants less than or equal to 34 weeks gestational age into the Microbiome, Atopic Disease, Prematurity (MAP) Study from two level III NICUs in San Diego County, California, USA. We examined a sub-cohort of infants born to mothers with a history of asthma and compared them to preterm infants with no maternal history of asthma. We analyzed stool and milk samples from three time points: at birth, 2 weeks, and 4-6 weeks postnatal age during their NICU stay. We then compared differences in bacterial community structure and metabolomic profiles between maternal asthma and control patients, as well as assessed a variety of clinical factors associated with their hospital care.

\section{Methods}

\section{Ethics Statement}

The University of California San Diego Institutional Review Board approved all study protocols (IRB approval \#181711). All research, experiments and data analysis were performed in accordance with University of California guidelines and regulations. Participant parents/guardians provided written, 
informed consent prior to enrollment in this research project. Furthermore, the study "The Association Between Milk Feedings in the Preterm Population, the Microbiome and Risk of Atopic Disease, MAP (Microbiome, Atopic Disease, Prematurity) Study" was registered in ClinicalTrials.gov database (NCT04835935).

\section{MAP Study Recruitment, Sample Collection, Outcome Measures, and Statistical Analysis}

We performed a prospective observational study at two level III NICUs in San Diego, California, USA from June 2019 to September 2020. Preterm infants born at 34 weeks gestational age or less were eligible for inclusion in this study. Infants were excluded if they were born with congenital anomalies that impacted the gastrointestinal system or those unable to follow up (i.e., infants born via surrogacy, infants' family reside in different cities). The MAP study sample size estimation was based on effect size for beta and alpha diversity differences, as previously described ${ }^{39}$. Utilizing effect size for beta diversity in Durack et al, we used a two-sample t-test, which implied a $n=16$ would give a power of 0.8 . Utilizing effect size for alpha diversity slope ( 0.14 vs. 0.22 over time between treatment groups) we calculated a one-way $\mathrm{K}$ means ANOVA which implied $n=8$ in each group would give a power of 0.8 . Considering that $30-50 \%$ of patients might be lost throughout the duration of our pilot study, we recruited a convenience sample size of 50 infants.

Samples were collected weekly from stool, milk feeds, and oral saliva. Stool samples were swabbed using BD Falcon ${ }^{\mathrm{TM}}$ SWUBE ${ }^{\mathrm{TM}}$ Dual Swab (cotton tip) from soiled diapers and meconium was prioritized in the first week of life, where possible. Milk feed samples were taken from the feeding syringe or bottle before or after feeding events. Milk feeds were recorded based on which milk type the preterm infant was receiving at the time of sample collection and included unfortified human milk, fortified human milk, and preterm formulas. Oral samples were collected by swabbing the buccal mucosa for five seconds using BD Falcon ${ }^{\mathrm{TM}}$ SWUBE ${ }^{\mathrm{TM}}$ Dual Swab (cotton tip). All collected samples were stored immediately after collection at $4^{\circ} \mathrm{C}$ and transferred to $-80^{\circ} \mathrm{C}$ within $12-36$ hours. Additionally, maternal demographic information was collected, including maternal dietary history during pregnancy, family history of atopic disease, and antenatal courses. Infant demographics collected included birth history and clinical course data (e.g., preterm morbidities, feeding history, and antibiotic usage).

The primary outcome of the MAP study is the clinical diagnosis of atopic diseases among patients after discharge from the NICU, including food allergies, allergic rhinitis, eczema, and asthma. The secondary outcome is the recognition of allergic sensitization patterns among the participants, measured by ImmunoCAP Multitest (ThermoFisher inc.), a blood test that provides qualitative responses (positive or negative) for food and environmental allergens.

Statistical analysis for clinical data was performed using GraphPad Prism 7 (Graphpad Software, Inc.). An unpaired Student-T test was used to analyze continuous clinical variables with a normal distribution. Either a Chi-square test or a Fisher's exact test was used to compare categorical variables, where 
appropriate. Mann-Whitney U-test was employed to analyze continuous variables with non-normally distributed histograms.

\section{Maternal Asthma Sub-Cohort}

For the pilot sub-cohort, we identified nine preterm infants born to mothers with a history of asthma (present or past), as well as a control group without maternal asthma diagnosis, of which paired infants were of the similar gestational age and sex. All 18 infants were born at the University of California, San Diego, Jacobs Medical Center. Meconium samples from birth and stool and milk samples from 2 and 4-6 weeks were analyzed for microbiome and metabolomic profiles. These time courses were chosen to include infants on minimal enteral feeds, full enteral feeds, and feeds taken orally. A total of 54 stool samples, including 18 meconium samples, and 36 milk feed samples were included in our analyses.

\section{Metagenomics}

DNA was extracted from samples using the Qiagen MagAttract PowerSoil DNA kit following the Earth Microbiome Project protocol ${ }^{45}$. Input DNA was quantified using a PicoGreen fluorescence assay (ThermoFisher, Inc) and library preparation was subsequently performed using a 1:10 miniaturized KapaHyperPlus protocol as previously described ${ }^{46}$. All samples were then normalized, based on a PicoGreen fluorescence assay, prior to sequencing on the Illumina NovaSeq platform (SP 300, PE150) at the Institute for Genomic Medicine (IGM), UC San Diego.

Raw metagenomic sequences were quality filtered and trimmed using fastp 47 , and human reads were filtered using minimap $2{ }^{48}$. Resulting reads were aligned using Bowtie2 and classified with the Woltka pipeline ${ }^{49}$ (Web of Life Toolkit App; v 0.1.1; https://github.com/qiyunzhu/woltka) against the Web of Life database ${ }^{50}$, resulting in a table of Operational Genomic Units (OGUs). Taxonomic profiles were analyzed in an R environment with the mctoolsr package (Leff, J. W. 2016. mctoolsr: microbial community data analysis tools. R package version 0.1.1.1. https://github.com/leffj/mctoolsr). Stool samples were rarefied to 14,000 reads, and breast milk samples were rarefied to 7400 reads for all downstream analyses. OGU richness was compared with Kruskal-Wallis. Additionally, differences in bacterial community composition were calculated among samples with Bray-Curtis dissimilarity ${ }^{51}$, weighted by OGU abundance and compared with a permutational multivariate analysis of variance (PERMANOVA), in which stool and milk samples were compared separately.

\section{Metabolomics}

For untargeted metabolomics by LC-MS, metabolomics extraction and data acquisition, using LC-MS was completed in the Collaborative Mass Spectrometry Center at UC San Diego.

Stool sample swabs were transferred to a 96-deepwell plate over ice, and metabolites were extracted with a solution of 1:9 LCMS grade water to ethanol. Swabs were incubated in the solvent overnight at $-20^{\circ} \mathrm{C}$ and removed from the wells after the incubation period. Breast milk samples were thawed and $50 \mathrm{uL}$ was 
transferred to a new eppendorf $2 \mathrm{~mL}$ tube over ice. Milk-associated metabolites were extracted by adding $100 \%$ LCMS grade methanol to each sample to create a 1:4 sample to methanol volume for extraction.

The extracted metabolites were concentrated down and resuspended in a 1:1 methanol to water (LCMS grade) solution. An ultrahigh performance liquid chromatography system (Thermo Dionex Ultimate 3000 UHPLC) coupled to an ultrahigh resolution quadrupole time of flight (qToF) mass spectrometer (Bruker Daltonics MaXis HD) was used for data acquisition. Metabolomics data processing and feature detection was completed using MZmine software (http://mzmine.github.io/). Library annotation and molecular networking was completed on the Global Natural Products Social Molecular Networking (GNPS) platform (https://gnps.ucsd.edu/ProteoSAFe/static/gnps-splash.jsp) feature based molecular networking workflow ${ }^{52,53}$. Data visualizations and machine learning analyses (random forest - sample classification) were completed using QIIME ${ }^{54}$ (https://qiime2.org/), and the Dunn's Test was run on specific metabolites that were identified to be significantly different between the maternal asthma and control groups using R scripts.

\section{Human Milk Oligosaccharides}

Concentrations of HMOs ( $\mathrm{mg} / \mathrm{mL})$ were measured, as previously described ${ }^{55}$. Briefly, 20 uL of human milk were dried in a 96-well plate and oligosaccharides were fluorescently labeled with 2-

aminobenzamide $\left(2 \mathrm{AB}\right.$, Sigma) in a thermocycler heat block at $65^{\circ} \mathrm{C}$ for exactly 2 hours. The reaction was stopped abruptly by reducing the thermocycler temperature to $4^{\circ} \mathrm{C}$. The amount of $2 \mathrm{AB}$ was titrated to be in excess to account for the high and variable amount of lactose and other glycans in milk samples. Labeled oligosaccharides were analyzed by HPLC (Dionex Ultimate 3000, Dionex, now Thermo) on an amide-80 column ( $15 \mathrm{~cm}$ length, $2 \mathrm{~mm}$ inner diameter, $3 \mu \mathrm{m}$ particle size; Tosoh Bioscience)) with a 50$\mathrm{mmol} / \mathrm{L}$ ammonium formate-acetonitrile buffer system. Separation was performed at $25^{\circ} \mathrm{C}$ and monitored with a fluorescence detector at $360 \mathrm{~nm}$ excitation and $425 \mathrm{~nm}$ emission. Peak annotation was based on standard retention times of commercially available HMO standards and a synthetic HMO library and offline mass spectrometric analysis on a Thermo LCQ Duo lon trap mass spectrometer equipped with a Nano-ESI-source. Concentrations were estimated by calculating area under the curve for each annotated HMO. Absolute quantification was not calculated because of fortifier interference.

\section{DATA AVAILABILITY}

Metagenomic raw sequence files and metadata tables are publicly available in the NCBI Sequence Read Archive (SRA; BioProject ID PRJNA750485), as well as in the Qiita repository (Study ID 13241).

Additionally, all R code used for metagenomic analyses are available on GitHub at https://github.com/hillms/MAP.

Metabolomic raw data, .mzXML files spectral files (.mgf) and feature quantification tables can be found on the MassIVE (https://massive.ucsd.edu/) database, under the accession number MSV000086246. The feature based molecular networking job can be found here:

https://gnps.ucsd.edu/ProteoSAFe/status.jsp?task=8a62828118e84fc39c30439425628627. 


\section{Results}

\section{MAP Patient Characteristics}

One hundred and seventy-seven eligible infants were identified in two level III NICUs, and 52 infants were recruited. Of these, six infants were later excluded prior to sample collection, resulting in 46 infants completing the NICU portion of the MAP study (Figure 1).

All mothers, 35 in total, received antenatal betamethasone. Seventy-seven percent of mothers had a Csection. Thirty-one percent of mothers had preterm rupture of membranes and received latency antibiotics. Eleven percent received antibiotics for chorioamnionitis. Twenty-three percent of mothers received antibiotics earlier during pregnancy for other reasons. In addition, most mothers received antibiotics preoperatively for C-section or Group-B streptococcus (GBS) prophylaxis for vaginal delivery during the intrapartum period. Overall, only one mother did not receive any antibiotics prior to delivery (Supplementary Table 1).

The average gestational age for the 46 infants in the cohort was 31.0 weeks, ranging from 23.3 weeks to 34.0 weeks. The average weight was $1591 \mathrm{~g}$, ranging from $720 \mathrm{~g}$ to $2290 \mathrm{~g}$. Forty-three percent of the infants were female. The majority of the infants were Caucasian (39\%), followed by Hispanic (22\%), and Asian (6\%). Sixteen infants (35\%) required intubation while 14 infants (30\%) received surfactant. Thirty percent of infants required systemic antibiotics for more than 2 days. Forty-two (91\%) infants experienced at least one episode of breastfeeding (nutritive or non-nutritive) during their NICU stay. Only 17 infants (40\%) were discharged home primarily receiving maternal breast milk (Supplementary Table 2).

\section{Characteristics of Maternal Asthma and Control Sub-Cohort}

For the sub-cohort maternal asthma analysis, the average birth weight in the control group was $1381 \mathrm{~g}$ compared to $1242 \mathrm{~g}$ in the maternal asthma group. Overall, patient characteristics were similar between the groups, including the average gestational age, birthweight, APGAR scores, pairs of twins, rate of Csection, maternal antibiotic exposure, and infant antibiotic exposure (Table 1).

Table 1: Clinical characteristics of the control and maternal asthma group. There are no statistical differences. 


\begin{tabular}{|c|c|c|}
\hline Characteristics & $\begin{array}{l}\text { Infant with maternal } \\
\text { history of asthma } \\
\mathrm{n}=9\end{array}$ & $\begin{array}{l}\text { Infant without maternal history } \\
\text { of asthma (Control) } n=9\end{array}$ \\
\hline Gestational Age, average weeks (range) & $29.7(24.1-34)$ & $29.6(24.3-32.6)$ \\
\hline Average weight, g (range) & $1242(575-2290)$ & $1381(605-2320)$ \\
\hline Male infants, $n$ & 5 & 2 \\
\hline C-section, $\mathrm{n}$ & 8 & 5 \\
\hline Prenatal Betamethasone, $\mathrm{n}$ & 9 & 9 \\
\hline Premature Rupture of membrane, $\mathrm{n}$ & 1 & 2 \\
\hline APGAR, average (Range) at $1 \mathrm{MOL}$ & $5(2-8)$ & $6(1-8)$ \\
\hline APGAR average (Range) at $5 \mathrm{MOL}$ & $8(3-9)$ & $7(3-9)$ \\
\hline Pair of Twins & 2 & 1 \\
\hline Family ownership of dog, $n$ & 2 & 6 \\
\hline Older siblings in house, $\mathrm{n}$ & 2 & 6 \\
\hline Current smoking in household, $\mathrm{n}$ & 2 & 1 \\
\hline Maternal antibiotics during pregnancy, $\mathrm{n}$ & 4 & 5 \\
\hline Maternal antibiotics during delivery, $\mathrm{n}$ & 9 & 7 \\
\hline $\begin{array}{l}\text { Infant received antibiotics during study } \\
\text { period }\end{array}$ & 7 & 4 \\
\hline $\begin{array}{l}\text { Infant with required antibiotics for more } \\
\text { than } 2 \text { days during study period }\end{array}$ & 4 & 4 \\
\hline Intraventricular hemorrhage & 3 & 2 \\
\hline ROP & 1 & 0 \\
\hline Necrotizing enterocolitis & 0 & 0 \\
\hline PDA required medical treatment & 1 & 2 \\
\hline Needing home oxygen & 1 & 0 \\
\hline Needing G-tube & 1 & 3 \\
\hline
\end{tabular}

Abbreviations: MOL: minute of life, ROP: retinopathy of prematurity, PDA: patent ductus arteriosus, Gtube: gastrostomy tube.

All infants received maternal milk or donor human milk, human milk fortified by bovine-based fortifier, or preterm formula based on the unit's feeding policy, infant's clinical status, and infant's corrected 
gestational age at the time of sample collection. For each subject, three longitudinal stool samples collected from birth (timepoint 1), 2 weeks of age (timepoint 2), 4-6 weeks of age (timepoint 3), and two longitudinal milk feed samples from timepoints 2 and 3 were analyzed. A total of 54 stool samples, including 18 meconium samples, and 36 milk feed samples were analyzed (Figure 2).

\section{Stool-Associated Bacterial Metagenome and Metabolome}

There were 2456 unique operational genomic units (OGUs; defined as sequence alignment hits to individual reference genomes) represented across all stool samples (ranging from 147 - 546 OGUs/sample). There was no observed association between maternal asthma diagnosis and bacterial richness or community composition in infant stool samples $(p=0.66, p=0.398)$. When assessing changes in bacterial profiles between the maternal asthma and control groups at different timepoints, we did not observe any differentiation between communities, except at timepoint $2(p=0.0481)$. However, this difference was not observed by the third timepoint $(p=0.841)$, suggesting that there are no significant differences in the preterm infant gut microbiome after 6 weeks of NICU exposure between infants with and without a maternal history of asthma.

Fecal microbial profiles revealed that bacteria in the genus Klebsiella, family Enterobacteriaceae, was highly abundant in both the maternal asthma and control groups across all timepoints $(35.4 \%$ and $22.0 \%$ of reads, respectively; $p=0.197$; Figure 3). In the maternal asthma group, Klebsiella represented $2.3 \%$, $56.6 \%$ and $42.5 \%$ of all reads at birth, 2 weeks, and $4-6$ weeks postnatal age, similar to what was observed at the same timepoints in the control group $(2.1 \%, 22.3 \%$ and $44.5 \%$ of all sequence reads). Further, the relative abundance of the genus Bacteroides sharply decreased in the maternal asthma group, by nearly $1 / 5$ of the total read count $(18.1 \%, 0.9 \%$ and $0.3 \%$ at timepoints $1,2,3)$, where this taxon stayed relatively stable and abundant over time in the control group $(15.4 \%, 19.2 \%$, and $15.1 \%$ at timepoint 1, 2, 3; Figure 3).

Bacteroides fragilis, whose early intestinal colonization in term infants is associated with increased risk of asthma ${ }^{27}$, only comprised $<0.5 \%$ of all reads recovered in our dataset. However, the relative abundance of Bacteroides at all timepoints, including at timepoint 3 only, were not significant between asthma and control groups ( $p=0.091$ at all timepoint, $p=0.19$ at timepoint 3$)$. Lastly, both maternal asthma and control group showed minimal abundances of bacteria from the families of Bifidobacteriaceae and Lactobacillaceae at less than $1 \%$ reads in stool samples from both groups.

When considering additional clinical factors, richness and community composition were significantly correlated with timepoint $(p=0.002, p=0.001)$ and infant antibiotic exposure $(p=0.009, p=0.011)$. In addition, there was a strong differentiation in bacterial community composition after the initiation of bottle $(p=0.014)$ and breastfeeding $(p=0.014)$, indicating changes to the stool microbiome at the onset of oral feeding, following full enteral feeds via a nasogastric tube. However, these differences were not apparent by timepoint 3 , in which there were no observed differences in bacterial community structure due to infant bottle feeding or breast feeding $(p=0.34, p=0.52)$. 
Metabolomic analysis of the stool samples showed significant differences between the maternal asthma group and the control group at 4-6 weeks postnatal age (timepoint 3; $p=0.027$ ) but not at earlier timepoints ( $p=0.81$ at timepoint $1, p=0.18$ at timepoint 2 ) (Figure $4 a-c, 4 e)$. The stool samples were classified between the maternal asthma group or the control group using a random forest sample classification analysis. The resulting ROC (Receiver Operating Characteristics) curve showed a high predictability of the maternal asthma group with an AUC (area under the curve) of 0.89 (Figure $4 \mathrm{~d}$ ).

Random forest analysis identified multiple metabolites in the linoleic acid network that were different between the maternal asthma group and the control group at the 4-6 week timepoint (Figure 5). Most linoleic acid metabolites represented in our samples were more abundant in the maternal asthma group, while a few showed an increase in abundance within the control group (Figure 5). In addition, stoolassociated metabolites differed based on infant antibiotic use $(p=0.001$, pseudo- $F=5.27)$, in agreement with the metagenomic data, but they did not differ after initiation of bottle or breast feeding $(p=0.07 ; p=$ 0.31). In the primary untargeted metabolomic analysis, lacto-n-fucopentaose (LNFP) isomer, a human milk oligosaccharide ( $\mathrm{HMO}$ ), was initially implicated in the machine learning differentiation between the maternal asthma group and the control group, but further analysis with a Kruskal-Wallis test did not show a statistical significance in LNFP isomer level in stool samples between the two groups at timepoint 3 ( $p$ $=0.12$ ).

\section{Milk Feed-Associated Bacterial Metagenome, Metabolome and Human Milk Oligosaccharides}

Milk feed samples were analyzed at timepoints 2 and 3 only, as most preterm infants did not receive enteral feed immediately after birth (timepoint 1 ). Milk feed compositions were similar between the maternal asthma and control groups ( $p=0.31$, Table 2$)$.

Table 2: Milk feed composition between the two groups. There is no statistical difference.

\begin{tabular}{|lll|}
\hline Milk Feed Composition & Maternal Asthma, $\mathbf{n = 1 8}$ & Control, $\mathbf{n = 1 8}$ \\
\hline Maternal milk & 1 & 3 \\
\hline Fortified maternal milk & 11 & 9 \\
\hline Fortified donor milk & 2 & 2 \\
\hline Fortified mixed maternal and donor milk & 1 & 0 \\
\hline Preterm formula & 1 & 4 \\
\hline Unknown & 2 & 0 \\
\hline
\end{tabular}

Among milk samples, OGU abundance ranged between 144 to 439 unique taxa per sample. Bacterial richness, bacterial community composition, and metabolite profiles were not correlated with any of the clinical variables tested in our study, including between maternal asthma and control groups (richness: $p$ $=0.663$, bacterial community composition: $p=0.369$ ). However, since there was initially evidence of 
differences between the maternal asthma and control groups for the HMO LNFP isomers in infant stool, and this $\mathrm{HMO}$ plays a role in supporting gut microbiome health, we examined the composition of the HMOs in the milk feed samples. HMO analysis of the milk feed samples from the two groups ( $n=16$ for control group and $n=11$ for maternal asthma group) showed no differences in LNFP 3 levels $(p=0.37)$, but there was a trend showing less LNFP 3 in the maternal asthma group (Figure 6).

\section{Discussion}

Here we present a unique paired dataset of bacterial communities and metabolites from stool and milk feed samples collected from preterm infants that are at risk for asthma development. We demonstrated that preterm infants born to mothers with a history of asthma showed different stool metabolomic profiles compared to those without a maternal asthma history, particularly at 4-6 weeks postnatal age. We did not observe a similar longitudinal change in the fecal bacterial communities; however, this could be due to small sample sizes and a short period of collection. For milk feed samples, we did not observe differences in the bacterial, metabolomic, or HMO profiles between the maternal asthma and control group.

The overall microbial profile from our stool samples were consistent with previous studies. Klebsiella, which belongs to family of Enterobacteriaceae, was the most abundant genus observed among fecal samples, while genera from families Bifidobacteriaceae and Lactobacillaceae were undetected or minimal, a pattern commonly observed in premature infants ${ }^{19,20,28}$. In addition, stool microbial patterns showed significant change over three timepoints, similar to prior studies ${ }^{14,19}$. When we quantified the impact of NICU exposures on the stool-associated bacterial community richness and composition, as well as the metabolome, there were differences associated with an infant's exposure to antibiotics at all timepoints, which is also consistent with previous studies ${ }^{29-31}$.

In comparing the stool-associated bacterial metagenomic patterns between maternal asthma and control groups, we observed a loss of Bacteroides in the maternal asthma group overtime, while the level remained stable in the control group. This is particularly notable as the abundance of Bacteroides bacteria in the gut provides a protective effect in the development of immune disease ${ }^{32-35}$. Our results showed a trend consistent with preservation of Bacteroides in the control group, but not in the maternal asthma group. Although it is not statistically significant, the pattern observed suggests that dysbiosis could begin as early as the neonatal period in populations at risk of atopic disease.

There is growing evidence that microbiome-metabolome interactions are involved in the immune system ontogeny and are important in asthma development ${ }^{23}$. Although maternal asthma history is known to increase an offspring's risk for developing childhood asthma, the mechanism is unclear and multifactorial ${ }^{1,2}$. However, our results might provide insight into asthma disease pathogenesis, in which the metabolomic analysis showed that compounds in the linoleic acid family differed in the stool samples between preterm infants born to mothers with a history of asthma compared to those without. Linoleic acid (18:2w6; cis, cis-9,12-octadecadienoic acid) is a diet derived polyunsaturated fatty acid 
(PUFA) whose derivatives are involved in cell signaling ${ }^{36}$. As the parent compound for the family of omega-6 PUFA, linoleic acid can give rise to inflammatory eicosanoids ${ }^{23,36}$. Linoleic acid metabolites have been implicated in steroid-resistant asthma, by causing steroid unresponsiveness in a mouse model of allergic airway inflammation ${ }^{37}$. Additionally, metabolomic analysis of serum from adult patients with asthma identified linoleic acid as being more abundant in severe asthma patients compared to healthy controls ${ }^{10}$. Similarly, infants with bronchiolitis who had greater abundances of linoleic acid in their nasopharyngeal samples were more likely to develop wheezing and asthma later in life ${ }^{38}$. Although microbes do not produce PUFAs, there is increasing evidence of PUFA interactions with human gut microbes in asthma pathogenesis ${ }^{23}$. One study showed that supplementation of the probiotic Lactobacillus rhamnosus GG in infants increased the level of stool omega-3 fatty acid, which is an antiinflammatory PUFA ${ }^{39}$. Our study found higher linoleic acid metabolites in the stool samples from preterm infants in the maternal asthma group, suggesting a pro-inflammatory process starting as early as the neonatal period among this at-risk population.

Lacto-n-fucopentaose (LNFP) isomers are human milk oligosaccharides (HMO) that exert immunological effects as prebiotic substrates for the gut microbiota ${ }^{40,41}$. High LNFP 3 levels in maternal breast milk were protective against the development of cow's milk protein allergy in infants ${ }^{42}$. We found that the maternal asthma group had lower levels of LNFP 3 in the milk feeds; however, they did not reach statistical significance.

There were many strengths in our study design. The MAP study combined the expertise of neonatology and allergy/immunology, in collaboration with labs dedicated to analyzing large microbiome and metabolomic datasets and performing HMO analyses. The MAP study cohort encapsulated robust maternal and infant clinical information corresponding to the microbial and metabolomic analyses of the stool and milk feeds. In addition, our sub-cohort selected for maternal asthma analysis was a singlebatch paired analysis of the microbiome and metabolome in stool and milk feed samples, with an additional HMO analysis of milk feed samples. This approach minimized batch effects and allowed for direct comparison and correlation of clinical, microbiome, metabolome and HMO data.

There are also several limitations to our study. First, the sample size of our pilot sub-cohort maternal asthma groups was small with only nine patients in each group, although there were 54 stool samples and 36 milk samples analyzed longitudinally. We were restricted by the number of mothers with a history of asthma, and future studies will aim to specifically recruit more mothers with a history of atopy. This might allow us to increase the statistical power and further distinguish the gut microbial and metabolomic patterns in preterm infants at risk for atopy. Additionally, the microbial and metabolomic findings in our sub-cohort could be an NICU specific phenomenon, so follow-up outpatient data will be important to consider when providing context to the clinical significance of our findings. In addition, because a preterm infant's gut microbiota is characterized by delayed colonization of microbes and low biodiversity ${ }^{43,44}$, our small sample size and limited duration of sample collection could contribute to the lack of distinction in fecal microbial profiles between two groups. A limitation in the HMO analysis was 
that milk feed samples were fortified with bovine-based fortifiers. Although this analysis accurately reflects the standard clinical practice of how preterm babies are fed in the study NICU, the bovine fortifier contains maltodextrin, a polysaccharide that is labelled together with HMOs and interferes with the quantification of absolute concentrations of HMOs. Although it would have been ideal to analyze unfortified milk feeds at the various timepoints, this would not have accurately replicated the interactions of fortified milk feeds with the gut microbiome in the preterm infant in a real world NICU. To improve HMO quantification, future studies should include milk sampling prior to the addition of bovine fortifiers.

In conclusion, our study demonstrated novel differences in stool metabolites between preterm infants born to mothers with and without asthma. These differences were observed longitudinally from birth to 4 - 6 weeks postnatal age, with those differences becoming more apparent over time. The linoleic acid metabolic network is known to play a role in the development of asthma during the critical window of immune development. Follow-up data from the MAP cohort in these same infants after discharge will longitudinally assess the allergic sensitization patterns at one year of age and development of asthma by 4-6 years of age to better understand the clinical implication of our results. Thus, with this study, we have laid the groundwork to potentially identify preterm infants in the NICU at risk for allergic sensitization and the development of asthma. This could lead to the development of therapeutic interventions during a critical window of immune development to reduce the risk of childhood asthma in this susceptible population.

\section{Declarations}

\section{ACKNOWLEDGEMENTS}

The authors would like to thank enrolled patients and their families for their participation in the study. The authors would also like to thank the nursing staff from both study NICUs for their assistance in sample collection. This work was supported by grants from UC San Diego Institute for Public Health (IPH) and the UC San Diego Center for Microbiome Innovation Pilot Project Grant. This work was funded in part by a MOMI (Mother-Milk-Infant) Seeds pilot grant from UC San Diego's Mother-Milk-Infant Center of Research Excellence (MOMI CORE). The support of the Family Larsson-Rosenquist Foundation is gratefully acknowledged.

\section{AUTHOR CONTRIBUTIONS}

SAL, SLL, SSB designed the study. SSB, DM, JK recruited the patients and collected the samples. SSB collected clinical data. MST, KCW, SH, SSB, AF processed the data and performed bioinformatic analyses. SSB, SLL, SAL, MST, and KCW wrote the manuscript. MST, BG, JAG, PCD, RK, SJS, LB provided conceptual advice and edited the manuscript. All authors have approved the final version of this manuscript.

\section{COMPETING INTERESTS}


The authors declare no competing interests.

\section{References}

1. Lim, R. H., Kobzik, L. \& Dahl, M. Risk for asthma in offspring of asthmatic mothers versus fathers: A meta-analysis.PLoS One5, (2010)

2. Mirzakhani, H. et al. Maternal asthma, preeclampsia, and risk for childhood asthma at age six. Am. J. Respir. Crit. Care Med, 200, 638-642 (2019).

3. Pascal, M. et al. Microbiome and allergic diseases.Front. Immunol.9, (2018)

4. Oddy, W. H. \& Breastfeeding Childhood Asthma, and Allergic Disease. Ann. Nutr. Metab, 70, 26-36 (2017).

5. Arrieta, M. C. et al. Early infancy microbial and metabolic alterations affect risk of childhood asthma.Sci. Transl. Med.7, (2015)

6. Huang, Y. J. et al. The microbiome in allergic disease: Current understanding and future opportunities -2017 PRACTALL document of the American Academy of Allergy, Asthma \& Immunology and the European Academy of Allergy and Clinical Immunology. J. Allergy Clin. Immunol, 139, 1099-1110 (2017).

7. Stokholm, J. et al. Maturation of the gut microbiome and risk of asthma in childhood. Nat. Commun, 9, 1-10 (2018).

8. Noto, A., Fanos, V. \& Dessì, A. Metabolomics in Newborns. Advances in Clinical Chemistry vol. 74 (Elsevier Inc., 2016)

9. Zhu, Z., Camargo, C. A. \& Hasegawa, K. Metabolomics in the prevention and management of asthma. Expert Rev. Respir. Med, 13, 1135-1138 (2019).

10. Reinke, S. N. et al. Metabolomics analysis identifies different metabotypes of asthma severity. Eur. Respir. J.49, (2017)

11. Barcik, W., Boutin, R. C. T., Sokolowska, M. \& Finlay, B. B. The Role of Lung and Gut Microbiota in the Pathology of Asthma., 52, 241-255 (2020).

12. Costello, E. K., Stagaman, K., Dethlefsen, L., Bohannan, B. J. M. \& Relman, D. A. The application of ecological theory towards an understanding of the human microbiome. Science (80-.), 336, 12551262 (2012).

13. Bäckhed, F. et al. Dynamics and stabilization of the human gut microbiome during the first year of life. Cell Host Microbe, 17, 690-703 (2015).

14. La Rosa, P. S. et al. Patterned progression of bacterial populations in the premature infant gut. Proc. Natl. Acad. Sci. U. S. A. 111, 12522-12527(2014)

15. Jost, T., Lacroix, C., Braegger, C. P. \& Chassard, C. New Insights in Gut Microbiota Establishment in Healthy Breast Fed Neonates.PLoS One7, (2012)

16. Palmer, C., Bik, E. M., DiGiulio, D. B., Relman, D. A. \& Brown, P. O. Development of the human infant intestinal microbiota. PLoS Biol, 5, 1556-1573 (2007). 
17. Song, S. J. et al. Cohabiting family members share microbiota with one another and with their dogs. Elife 2013, 1-22 (2013)

18. Werner, E. et al. Mode of delivery and neonatal outcomes in preterm, small-for-gestational-age newborns: Editorial comment. Obstet. Gynecol, 120, 560-564 (2012).

19. Korpela, K. et al. Intestinal microbiota development and gestational age in preterm neonates. Sci. Rep, 8, 1-9 (2018).

20. Underwood, M. A., Mukhopadhyay, S., Lakshminrusimha, S. \& Bevins, C. L. Neonatal intestinal dysbiosis. J. Perinatol, 40, 1597-1608 (2020).

21. Wikoff, W. R. et al. Metabolomics analysis reveals large effects of gut microflora on mammalian blood metabolites. Proc. Natl. Acad. Sci. U. S. A. 106, 3698-3703(2009)

22. Dodd, D. et al. A gut bacterial pathway metabolizes aromatic amino acids into nine circulating metabolites., 551, 648-652 (2017).

23. Lee-Sarwar, K. A., Lasky-Su, J., Kelly, R. S., Litonjua, A. A. \& Weiss, S. T. Gut microbial-derived metabolomics of asthma. Metabolites10, (2020)

24. Zaiss, M. M. et al. The Intestinal Microbiota Contributes to the Ability of Helminths to Modulate Allergic Inflammation., 43, 998-1010 (2015).

25. Tan, J. et al. Dietary Fiber and Bacterial SCFA Enhance Oral Tolerance and Protect against Food Allergy through Diverse Cellular Pathways. Cell Rep, 15, 2809-2824 (2016).

26. Trompette, A. et al. Gut microbiota metabolism of dietary fiber influences allergic airway disease and hematopoiesis. Nat. Med, 20, 159-166 (2014).

27. Vael, C., Nelen, V., Verhulst, S. L., Goossens, H. \& Desager, K. N. Early intestinal Bacteroides fragilis colonisation and development of asthma. BMC Pulm. Med, 8, 1-6 (2008).

28. Underwood, M. A. \& Sohn, K. The Microbiota of the Extremely Preterm Infant. Clin. Perinatol, 44, 337339 (2017).

29. Wandro, S. et al. The Microbiome and Metabolome of Preterm Infant Stool Are Personalized and Not Driven by Health Outcomes, Including Necrotizing Enterocolitis and Late-Onset Sepsis. mSphere 3(2018)

30. Russell, J. T. et al. Antibiotics and the developing intestinal microbiome, metabolome and inflammatory environment in a randomized trial of preterm infants. Sci. Rep, 11, 1-16 (2021).

31. Cuna, A., Morowitz, M. J., Ahmed, I., Umar, S. \& Sampath, V. Dynamics of the preterm gut microbiome in health and disease. Am. J. Physiol. - Gastrointest. Liver Physiol, 320, G411-G419 (2021).

32. Wexler, H. M. \& Bacteroides The good, the bad, and the nitty-gritty. Clin. Microbiol. Rev, 20, 593-621 (2007).

33. Wexler, A. G. \& Goodman, A. L. An insider's perspective: Bacteroides as a window into the microbiome.Nat. Microbiol.2, (2017)

34. Troy, E. B. \& Kasper, D. L. Beneficial effects of Bacteroides fragilis polysaccharides on the immune system. Front. Biosci, 15, 25-34 (2010). 
35. Neis, E. P. J. G., Dejong, C. H. C. \& Rensen, S. S. The role of microbial amino acid metabolism in host metabolism. Nutrients, 7, 2930-2946 (2015).

36. Whelan, J. \& Fritsche, K. Linoleic Acid 1. Adv. Nutr, 4, 311-312 (2013).

37. Panda, L. et al. Linoleic acid metabolite leads to steroid resistant asthma features partially through NF-KB. Sci. Rep, 7, 1-11 (2017).

38. Zhu, Z. et al. Metabolome subtyping of severe bronchiolitis in infancy and risk of childhood asthma. J. Allergy Clin. Immunol, 1-11 https://doi.org/doi:10.1016/j.jaci.2021.05.036. (2021).

39. Durack, J. et al. Delayed gut microbiota development in high-risk for asthma infants is temporarily modifiable by Lactobacillus supplementation.Nat. Commun.9, (2018)

40. Triantis, V., Bode, L. \& van Neerven J. R. J. Immunological effects of human milk oligosaccharides. Front. Pediatr. 6, (2018)

41. Davis, E. C., Wang, M. \& Donovan, S. M. The role of early life nutrition in the establishment of gastrointestinal microbial composition and function., 8, 143-171 (2017).

42. Seppo, A. E., Autran, C. A., Bode, L. \& Järvinen, K. M. Human milk oligosaccharides and development of cow's milk allergy in infants. J. Allergy Clin. Immunol, 139, 708-7115 (2017).

43. Henderickx, J. G. E., Zwittink, R. D., Van Lingen, R. A., Knol, J. \& Belzer, C. The preterm gut microbiota: An inconspicuous challenge in nutritional neonatal care. Front. Cell. Infect. Microbiol, 9, 1-12 (2019).

44. Rougé, C. et al. Investigation of the intestinal microbiota in preterm infants using different methods., $16,362-370$ (2010).

45. Marotz, C. et al. DNA extraction for streamlined metagenomics of diverse environmental samples., 62, 290-293 (2017).

46. Sanders, J. G. et al. Optimizing sequencing protocols for leaderboard metagenomics by combining long and short reads. Genome Biol, 20, 1-14 (2019).

47. Chen, S., Zhou, Y., Chen, Y., Gu, J. \& Fastp An ultra-fast all-in-one FASTQ preprocessor., 34, i884-i890 (2018).

48. Li, H. Minimap2: Pairwise alignment for nucleotide sequences., 34, 3094-3100 (2018).

49. Zhu, Q. et al. OGUs enable effective, phylogeny-aware analysis of even shallow metagenome community structures. bioRxiv 2021.04.04.438427 (2021)

50. Zhu, Q. et al. Phylogenomics of 10,575 genomes reveals evolutionary proximity between domains Bacteria and Archaea.Nat. Commun.10, (2019)

51. Bray, J. R. \& Curtis, J. T. An Ordination of the Upland Forest Communities of Southern Wisconsin. Ecol. Monogr, 27, 325-349 (1957).

52. Wang, M. et al. Sharing and community curation of mass spectrometry data with Global Natural Products Social Molecular Networking. Nat. Biotechnol, 34, 828-837 (2016).

53. Nothias, L. F. et al. Feature-based molecular networking in the GNPS analysis environment. Nat. Methods, 17, 905-908 (2020). 
54. Bolyen, E. et al. Reproducible, interactive, scalable and extensible microbiome data science using QIIME 2. Nat. Biotechnol, 37, 852-857 (2019).

55. Seferovic, M. D. et al. Maternal diet alters human milk oligosaccharide composition with implications for the milk metagenome. Sci. Rep, 10, 1-18 (2020).

\section{Figures}

\section{Figure 1}

MAP study recruitment
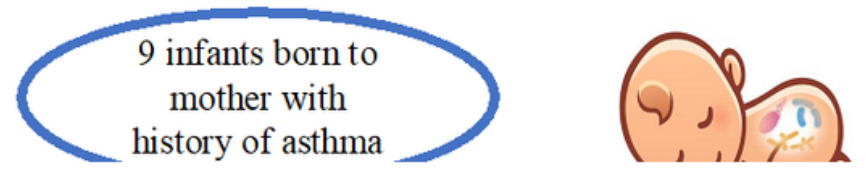

\section{Figure 2}

Maternal asthma pilot analysis study design schematic 


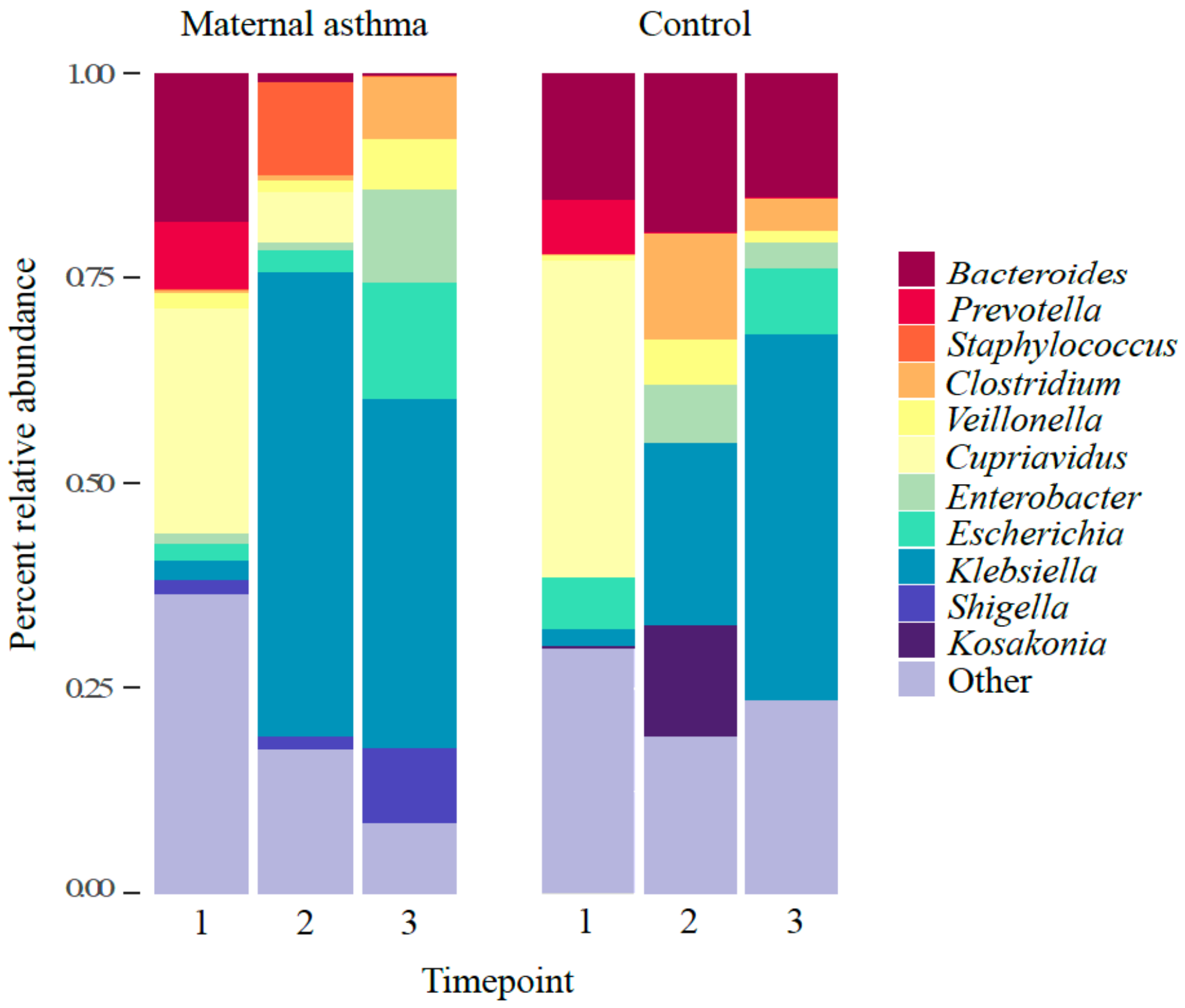

Figure 3

Fecal microbiome longitudinal changes between maternal asthma and control group based on shotgun sequencing. 
a.

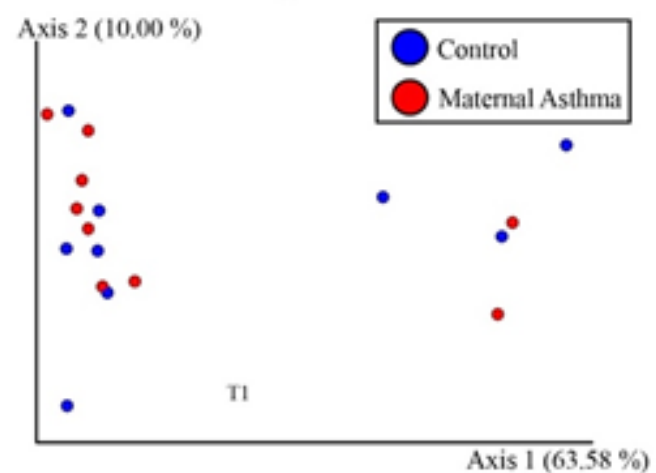

c.

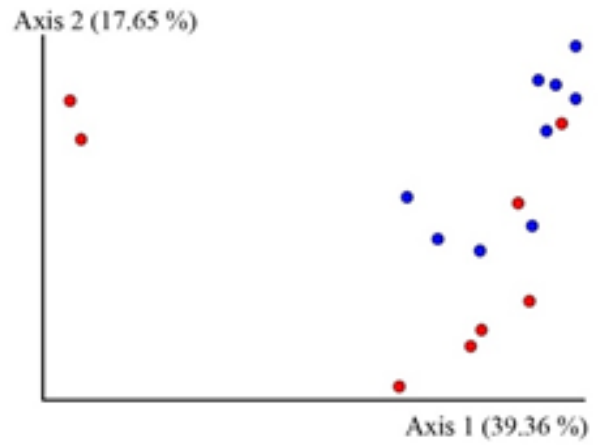

b.

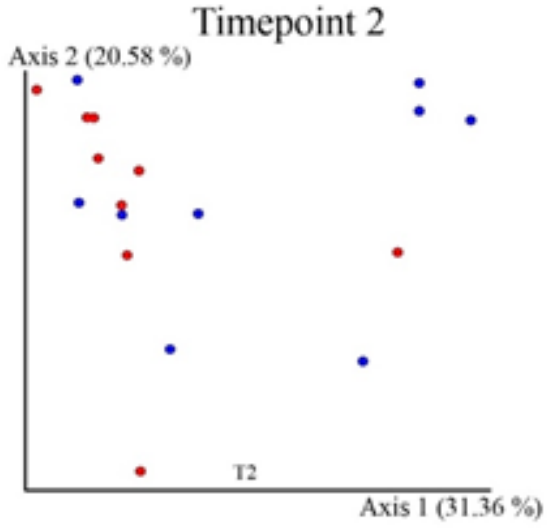

d.

Timepoint 3 - Per-Class Receiver Operating Characteristics

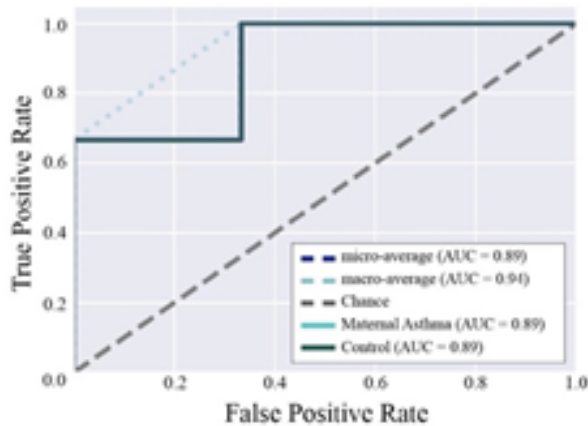

e.

\begin{tabular}{|l|l|l|l|}
\hline & Timepoint 1 (Birth) & Timepoint 2 (2 weeks) & Timepoint 3 (4-6 weeks) \\
\hline p-value & 0.81 & 0.18 & 0.027 \\
\hline pseudo-F & 0.42 & 1.37 & 2.33 \\
\hline
\end{tabular}

Figure 4

Fecal metabolomic profile between maternal asthma and control group. Metabolomic profiles differentiate over time and became statistically significant at the third timepoint. 


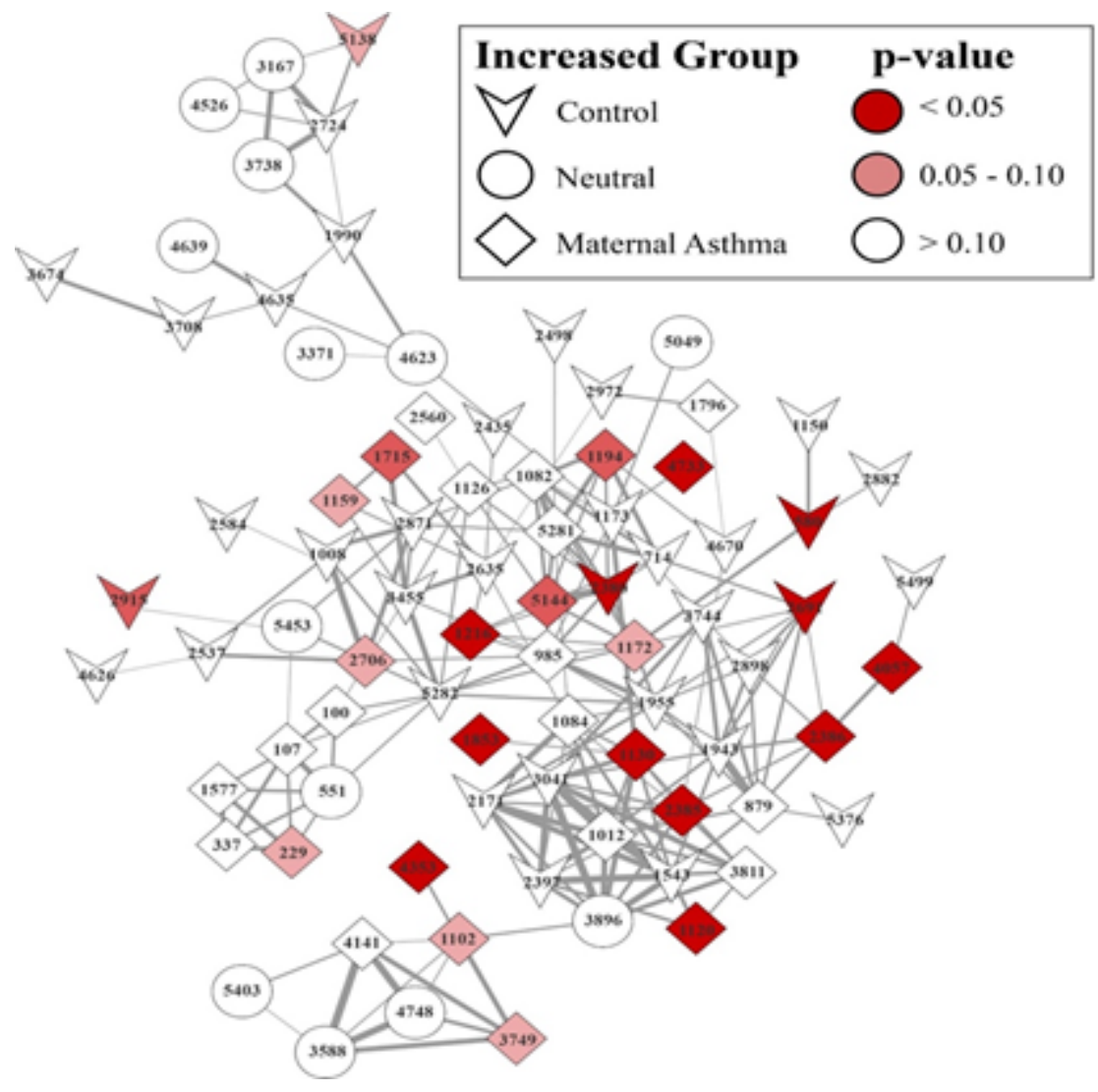

Figure 5

Fecal linoleic acid network at timepoint 3 (4-6 weeks postnatal age). Multiple chemicals in the linoleic acid network differ significantly between the maternal asthma group and control group. The shape indicates in which group the compound is increased. The coloring indicates if this increase is significant based on a Kruskal-Wallis test. The widths of the lines connecting the compounds are determined by the spectral similarity cosine score, with the widest line being a score of 1. Compound annotation (from GNPS library search) is provided in Supplementary Table 3.
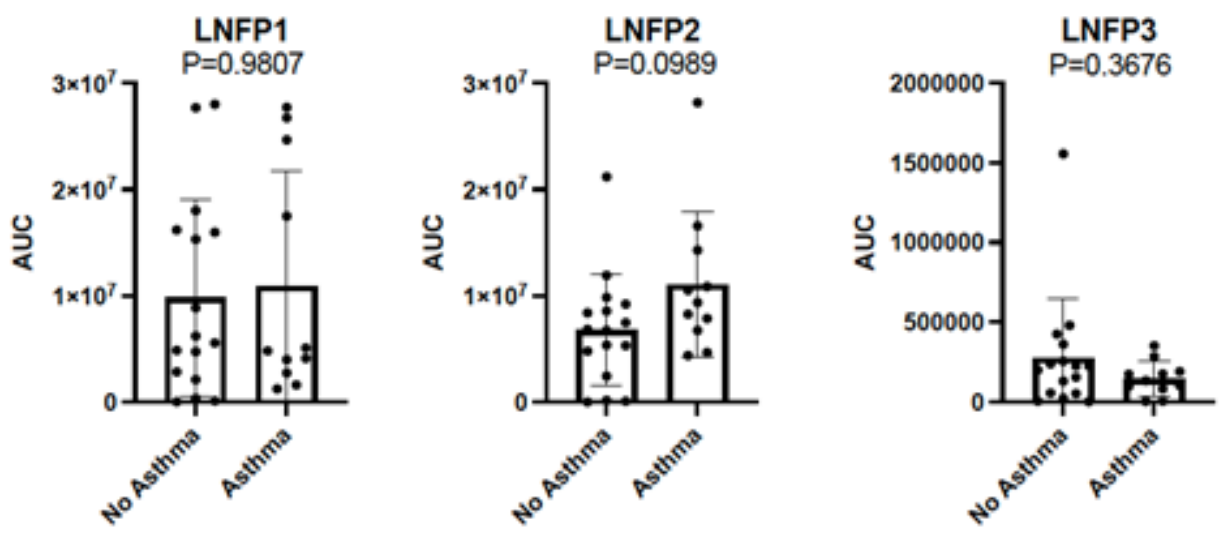

Figure 6 
$\mathrm{HMO}$ analysis of milk feed samples $\mathrm{HMO}$ analysis of milk feed samples. No significant difference was detected.

\section{Supplementary Files}

This is a list of supplementary files associated with this preprint. Click to download.

- MAPStudySupplementaryTables.docx 